\title{
A Novel Web-enabled Healthcare Solution on HealthVault System
}

\author{
${ }^{1}$ Lingxia Liao, ${ }^{12}$ Min Chen, ${ }^{1}$ Son Vuong, ${ }^{2}$ Xiaorong Lai \\ ${ }^{1}$ Dept. of Computer Science, Univ. of British Columbia, V6T 1Z4, Canada \\ ${ }^{2}$ WISENET Lab, Hebei Polytechnic University, China \\ Email: \{liaolx,vuong\}@cs.ubc.ca, minchen@ieee.org, roy.lai@ hotmail.com
}

\begin{abstract}
Complicated Electronic Medical Records (EMR) systems have created the problems in system easy implementation and interoperability for a Web-enabled Healthcare Solution, which is normally provided by an independent healthcare giver with limited IT knowledge and interests. An EMR system with well-designed and user-friendly interface, such as Microsoft HealthVault System, as the back end platform of a Web-enabled healthcare application will be an approach to deal with these problems. In this paper, through analyzing the patient oriented Web-enabled healthcare service application as the new trend to delivery healthcare from hospital/clinic-centric to patient-centric, the current e-healthcare applications, and the main backend EMR systems, we present a novel web-enabled healthcare solution based on Microsoft HealthVault EMR system to meet customers' needs. A sample system is given to show how this model can be used.
\end{abstract}

\section{INTRODUCTION}

Electronic healthcare (e-Healthcare) systems have been evolved for decades with the traditional healthcare providers such as hospitals, medical centers, laboratories and doctors' offices appearing. With a hospital-centric healthcare model, our traditional healthcare facilities are focused on how to meet healthcare providers' needs and how to manage doctors and other health staff [1]. Therefore, we have hospital management systems, billing systems, scheduling systems and so on. However, this "face-to-face" way for accessing to healthcare services has been changed a lot with the great evolution of Internet technology. Then, the Web-enabled healthcare service is emerging as a new healthcare delivery trend, increasing and empowering "e-patients" or "e-Customers" to seek healthcare information and purchase healthcare services through the Internet in an effective cost and efficient way.

According to the Wall Street analysts [4], the recent webenabled healthcare delivery industry has been fostered to be a market. However, when we are using "e-Healthcare" to search the Internet, we can hardly find the real online healthcare services except for websites of selling vitamins and books, etc. There is a big gap between the limited number of healthcare service providers and huge number of consumers. The main reason is that most of the existing e-Healthcare back-end platforms, EMR Systems, are too complicated for those Webenabled healthcare service providers, who normally are the independent healthcare professionals. They are expected to donate more time on illness curing rather than participating an IT project programming. Finding a well designed EMR plat- form with easily understood and used programming interface, developing a understandable and rapidly implementable Webenabled system model with good security, interoperability and low maintenance cost for those independent healthcare givers are the key point to deal with this trouble. In this paper, through summarizing the two-layered Web-enabled application infrastructural model and comparing the main back end EMR systems, we propose a novel Web-enabled healthcare solution by using Microsoft HealthVault EMR[7] system to show how it deals with the trouble in rapid implementation, interoperability, security and maintenance.

The rest of the paper is organized as following. Section 2 describes the background about healthcare information systems and gathers contributions for the proposed Web-enabled healthcare solution, such as, the two-layered infrastructure model, the main back-end EMR systems, and the customers' applications. On section 3, through analyzing healthcare application users' requirements, a novel Web-enabled healthcare solution on top of Microsoft HealthVault EMR back-end system is proposed. Section 4 presents a sample system based on the prosoed model and the conclusions are drawn on Section 5.

\section{BACKGROUND}

As we know, Canadian healthcare system is a publicly funded system and it can be split into two levels, the Primary healthcare services based on clinics and the Secondary services based on hospitals [2]. The major health care services and the way they are delivered have been changed from a reliance on hospitals and doctors to alternative care in clinics, primary health care centers, community health centers and so on [3], [4]. Normally, these health care providers are working independently and operating privately. It is almost impossible for them to invest huge amount of money to hire a professional development team with health care domain experience, which can be very expensive and prefer customizing their existing system rather than build a new one according to the new requirements; or to invest big amount of time to work with programmers. These are the main trouble that blocks the Webenabled health care services being delivered. A time and cost effective solution with necessary security and interoperability will be their best choice. On the rest of this section, we elaborate a two-layered infrastructure model, the main backend EMR platforms, and the customers' application on the 
front.

\section{A. The two-layered infrastructure model}

In order to provide Web-enabled healthcare solutions for millions of e-Patients, a two-layered model can be provided to turn them a reality. This model may consider the E-healthcare back-end platform layer and the e-Healthcare customer applications layer. This infrastructure can ensure that medical givers can devote more time to their patients, patients' health records can be accessed safely, and the healthcare services can be delivered more efficiently with lower costs and shorter wait times. Figure 1 displays the structure of this two-layered model.

The e-Healthcare customer's applications can be either the applications used in hospitals and clinics or the Web-enabled e-Healthcare services desired by millions of e-Patients. The former applications can be accessed through a local networking or the Internet by the authenticated users, such as, doctors or staff working in hospitals or clinics. The latter normally deployed through the Internet and work online. E-Patients are able to access to them through the Internet. Both of these two kinds of e-Healthcare applications must connect to the e-Healthcare back-end platform based on the EMR system, which electronicalizes all the private and sensitive medical information and provides some related services[10].

\section{B. The e-Healthcare back-end platform}

The e-Healthcare back-end platform is based on EMR systems, which is a longitudinal record of patient health information generated by one or more encounters in any care delivery setting[9]. Patient demographics, progress notes, problems, medications, vital signs, past medical history, immunizations, laboratory data and radiology reports are all included in it. It forms the platform to support the daily clinical workflow and the other care-related activities directly or indirectly via its interface. This interface sets the standards for all customers' applications on the top. Following, several available solutions are described and discussed in the context of the goal of this contribution.

1) Enterprise EMR systems [13]: Nowadays, many companies provide proprietary EMR systems for hospitals and clinics. As Dorenfest[18] and HIMSS [18] Analytics have tracked since 2002[13], the major enterprise/EMR vendors, for example, Medical Information Technology, Inc (MEDITECH) , Siemens Medical Solutions, McKesson Provider Technologies, Cerner Corps. , had got $88.82 \%$ of the market in 2005. They provide complex and comprehensive EMR systems for those big Hospitals. These EMR systems are normally developed on legacy system and have totally different interfaces,and they can be very expensive to evolve and very low chance to be interoperable. On the other hand, some small venders respect to those top ones we just mentioned, such as, eClinicalWorks[15], MedComSys[16] and Medinformatix[17], provide some EMR systems with small scale and flexible functionality to those medium/small/solo medical practices. As the same as the complecated ones, they also have the problems in interoperability[14] and the huge cost in system maintenance and redevelopment.

2) Free/Open EMR systems: In order to deal with the high cost and interoperability of EMR systems, the open source community has developed the free EMR system named the OSCAR[8]. This EMR system includes the full billing capabilities, chronic disease management tools, prescription module, scheduling and many other features. It can run locally in an office or be accessed through the Internet. The main benefits for users to choosing this free EMR system are described as the following:

- Affordability, total cost of ownership of the OSCAR EMR system and its data is usually dramatically less than proprietary EMR systems. The only fee we have to consider for building an OSCAR EMR system is to buy a suitable server box, the installation services, and/or monthly maintenance services.

- Sustainability, costs of proprietary products are expected to dramatically escalate over the next several years as the industry moves to narrow the vendor field and increases system requirements. This will create an ever more captive consumer market with proprietary systems and for those whose systems are not compliant with newer standards, it may mean a significant loss of investment and data.

However, OSCAR also presents three main disadvantages to turn it more used widely. Firstly, getting starting use and know OSCAR system for a doctor without full IT knowledge is very time consuming. There are no organizations or companies to provide pre-sale services to doctors and independent developers, the OSCAR website is the only way to get the related information. Secondly, even with the help of IT engineers, system customization is still pretty difficult. The OSCAR system is driven by the open source community and developed by millions of different developers, then the source code is piling up without full documentations and the system structure is getting complicated. Finally, it is impossible for an independent developer without any OSCAR developing experience to add new features in it within a short period. The services provided are limited regarding installation and maintenance, and there are few people understanding the system programming and can be dependable at all times.

3) Microsoft HealthVault EMR system: Microsoft HealthVault[7] is a system in the middle of the both above-mentioned. It is a healthcare back-end platform based on EMR system, plus a set of services for creating an application ecosystem that lets consumers collect, store, and share health information online. This well organized and maintained platform provides a privacy-enhanced and security-enhanced foundation that can be used to store and transfer information between a variety of e-healthcare customer's applications, hospital applications and healthcare devices.

This system has three major advantages. First, it presents a low cost solution in developing and maintaining. Contrast to the first two platforms that users have to purchase or 


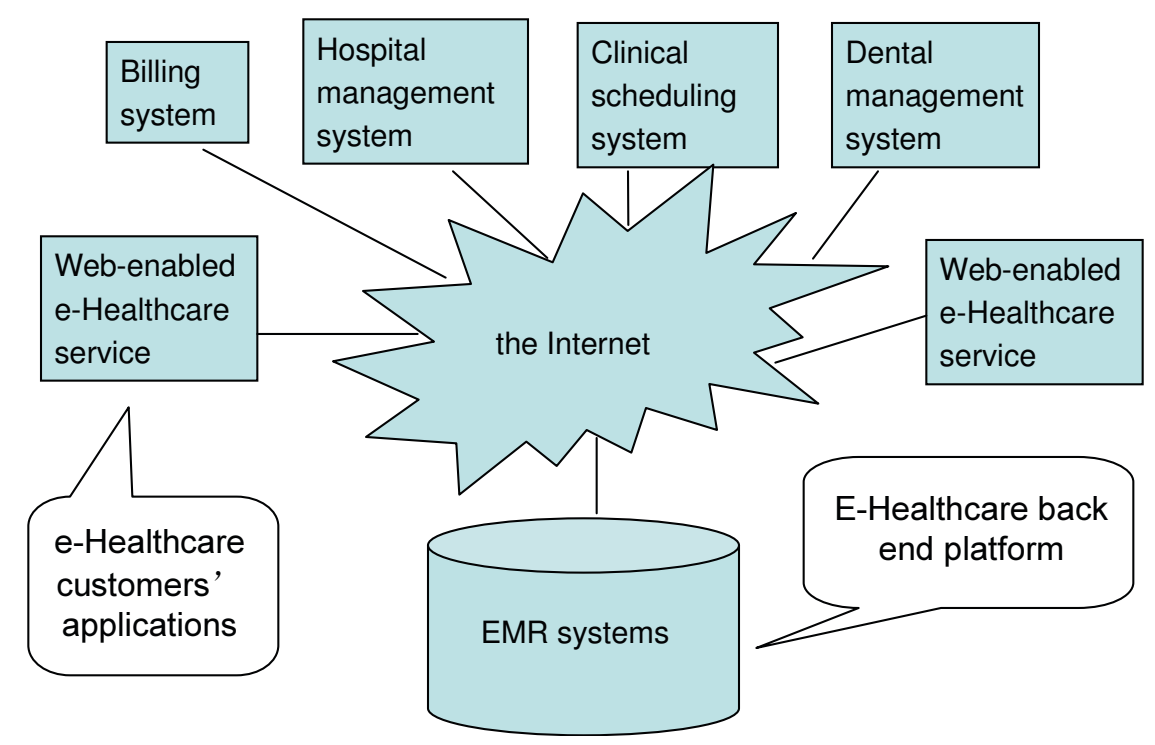

Fig. 1. E-Healthcare two-layered infrastructure model.

download a EMR copy, to get the suitable hardware, and to worry about the network bandwidth, database performance and access security, the HealthVault EMR system is always running somewhere of Microsoft datacenter with probably the best IT engineers in the world taking care of their security and performance. The only pay, which is much more less than purchasing software, hardware, and maintenance, is the joining fee for becoming a HealthVault application. Second, it is finely designed and used advanced technology. HealthVault system is the latest EMR system among the three we have mentioned above. It is designed by Microsoft experts and adopts the newest software engineering, database, information security, networking communication, and hardware technologies, which ensure that millions of front-end applications (the e-Healthcare customer's applications) can be successfully supported. The HealthVault team is still working very hard to improve the whole system and add more features, better performance and higher security can be expected to be coming. Finally, the system offers an easy customization. With sufficient documents and sample codes in Microsoft MSDN website[7], any interested individual with some experience in programming language $\mathrm{C \# [6]}$ and ASP.NET[7] can develop a customer application on top of HealthVault. New development solutions and models are also published on MSDN or through yearly conference. Online discussion can also be accessible on HealthVault developing forum.

\section{The e-Healthcare customers' applications}

All the healthcare applications on top of the EMR platform can be named as e-Healthcare customer's applications. They can be divided into two categories, the hospital/clinicaloriented and the e-patient-oriented. Hospital/clinical-oriented applications are traditional and have been involved for decades. Almost all the hospital healthcare system and part of clinical healthcare system in developed countries can be categorized into this sector[13]. They are legacy and cost a lot in updating and maintaining. More than $80 \%$ of them are based on proprietary EMR systems[13]. The interoperability has become one of the biggest issues[14]. E-patient-oriented applications are also called Web-enabled healthcare services. In recent years, they are merging as the result of health care delivery changing from hospital-centric to the Internet-based3. They also can be subdivided into two types, one for health care consumers to manage their own healthcare records and the other for health care givers to provide healthcare services. MyHealthVault[7] and MyOscar[8] provided by Microsoft and open source community belong to the first sector. Individuals can logon them and review/manage their health records. The Web-enabled services provided by healthcare providers are the most needed not only by e-Patients but also by the wellness concerned. Fully closed and opened back-end platform are too complicated for common healthcare actors such as family doctors, dentists, and pharmacists, to build their own Webenabled healthcare services. Microsoft HealthVault system can be a good choice with low cost, necessary security, and rapidly development tools to create a small and middle sized Webenabled healthcare service.

\section{Web-enabled HealthCare Solution on HEALTHVAult EMR SYSTEM}

As above-mentioned, the ways that people access to healthcare services have been changed from "face-to-face" to "through the Internet", and the ways of healthcare delivery have been changed from "hospitals/clinics-centered" to "patients-centered". Trillions of "e-Patients" or "wellnessconcerned" are looking for healthcare services online, but they barely can find them[4]. The main reason is that most of the EMR systems widely used today as the core back-end platform for front-end healthcare applications are those products based on previous trends. They put their focus on hospitals and 




Fig. 2. The working flow of the proposed solution.

clinics rather than patients. Such kind of the back-end platform has complicated structure and the interface is not shared. There is hardly a way to evolve it to the new trend, "patient-centric" web-enabled healthcare services, which normally needs to do the system redevelopment according to the EMR platform interface and provided by independent healthcare providers. In the rest of this sector, a novel Web-enabled healthcare solution based on HealthVault EMR platform will be discussed through analyzing the system requirements and the system design.

\section{A. System requirements}

The common Web-enabled healthcare services are provided by independent healthcare providers, and facing the wellness and patients concerned. So such kind of applications is normally small application, and its providers and consumers are all individuals, who may or may not have IT technology and programming background. Such kind of applications has several features.

- Cost effective. Since this kind of applications is small, and its owners and consumers are individuals, we can not expect them to spend huge money in building such kind of application or buying a service through it.

- User friendly. The user interface must be extremely friendly and easy to use. Both services providers and consumers are not IT technical oriented, and the most important is that we may be able to train the providers but never have chance to train our consumers, once they get confused, they never come back again.

- Easily developing, deploying and maintaining. As we above-mentioned, the providers are individuals, they may not have deep IT technology. The typical case is that they hire a couple of helpers to do the developing, deploying and maintaining. With the limited budget, we can not expect the helpers have deep domain knowledge and comprehensive system design skills. A simple system design, rapidly development model, less work in deploying and maintaining are what they want.

- Information security. Any information related to individual's healthcare record is privacy and sensitive. How this information is stored and accessed should be privacyenhanced and secure-enhanced is a big concern. In addition, customers might need to access to such kind of Web-enabled healthcare services to buy a healthcare service, the information about online paying also should be privacy-enhanced and secure-enhanced.

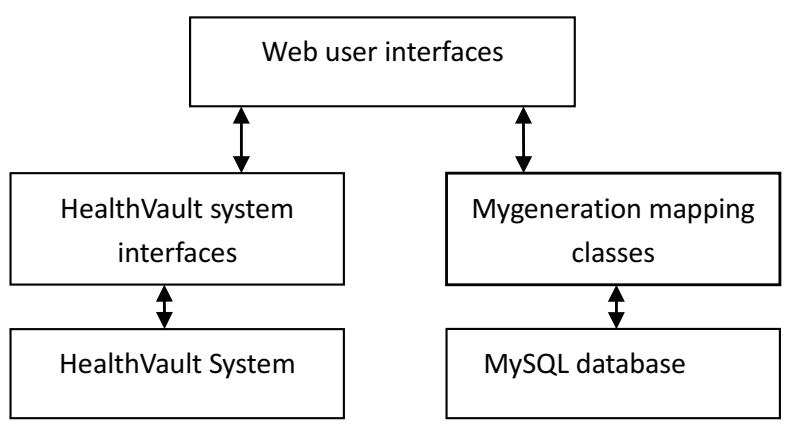

Fig. 3. The architecture design of the proposed web-enabled healthcare service solution.

Based on related literature and these system requirements, next section will focus on proposed system design.

\section{B. System design}

As discussed in previous sections, a Web-enabled healthcare service must be built on top of the EMR systems. In this proposed solution, we are going to employ Microsoft HealthVault EMR system instead of any other existing EMR systems used in hospitals or clinics because of its low cost, sophisticated architecture and enhanced security. The whole solution involves Microsoft Internet Information Server (IIS)[20] web server, Microsoft HealthVault system[21], and MySq1[22] database where some necessary help information may be stored locally. The workflow of our solution is shown in Figure 2.

In this system, we have two types of users, (i) healthcare customers and (ii) healthcare givers. Healthcare customers may go to our web pages to browse the free healthcare information as well as login to web-enabled healthcare services to purchase some healthcare advances, such as pharmacy consultant, weigh-lose guidance, fitness advances, or chronic disease watch. Healthcare givers can review the healthcare information, which has shared by its owner and give some answers through our system. The main functions include the following main functionalities: (1) users login and logout; (2) healthcare information share/retrieve/update/delete for login customers; (3) Shared healthcare information retrieve, advices give for login givers; and (4) bill paying. The whole system architecture is depicted in Figure 3.

As the back-end platform, HealthVault system works as a database to store all the EMR information in a proper way in which any user can not only add/update/delete/retrieve their own records but also share the records to the healthcare providers. The HealthVault system interface APIs are such kind of ways to be used to interact with HealthVault EMR system. All the APIs are programming in C\#[21], so all the web pages on the top must be written in ASP.NET/C\#[21] to easily call the APIs. Beside HealthVault system in the back-end, we also can find Mysql database in Figure 3. It is used for storing the user information and HealthVault visiting information so that only the authenticated customers can use the healthcare services and only those records shared by a authenticated customer can be visited by the healthcare 


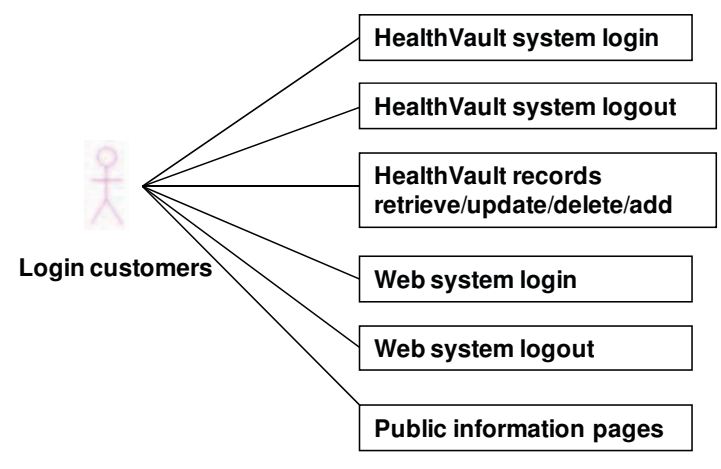

Fig. 5. the basic functionalities for login customers.

provider who are given the right by that authenticated customer through this proposed application. In this proposed design, we suggest to use Mygeneration[23], which is a free database mapping tool, to map MySQL tables into classes to avoid from writing the boring SQL[22] sentences and their testing.

From all the above-mentioned, a rapidly implementable and cose effective design has been proposed. By using HealthVault EMR system as the back-end platform, application developers inherit a free EMR platform with open interfaces, sample codes, documents, and even sample projects. The MySQL database and Mygeneration mapping tool are also free and save lots of energy for developers in database testing. C\# and ASP.NET programming languages are very handy and understandable for all developers with basic programming skills. Next section will present a sample system to show how the proposed design is implemented.

\section{SAMPLE SySTEM IMPLEMENTATION}

Using the proposed approach, we have implemented a Webenabled pharmacy service which provides pharmacy related consultants online. Figure 4 shows the sample system architecture. This sample system has multiple web pages including public pages and private pages. The public pages contain some general information about healthcare news, event, and the service provider. They can be browsed by anybody. The private pages consisting of the information related to a specific customer can only be accessed to by that login customer. The HealthVault records pages as a part of the private pages containing all the sensitive healthcare information have to be accessed to through the cutomer's live account. As abovementioned in the previous section, the whole system runs on top of HealthVault EMR platform and MySQL database. HealthVault EMR APIs and Mygeneration mapping classes are used to interact with the back-end EMR system and database to save developing and testing time.

Figures 5, 6, 7 depict the basic functionalities for each type of users. As above-mentioned in the previous section, there are two types of users, customers (public or login) and doctors (login). The public customers can browse the free healthcare information through the web page. The login customers can review/modify/share their private healthcare records stored in HealthVault system through our system and ask for healthcare

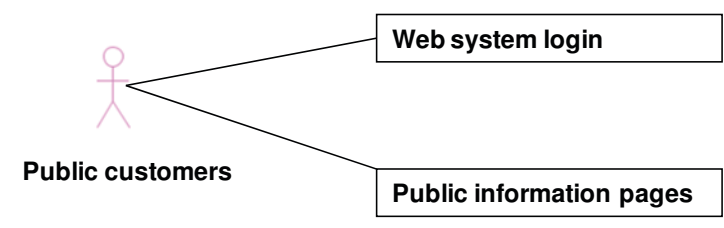

Fig. 6. the basic functionalities for public customers.

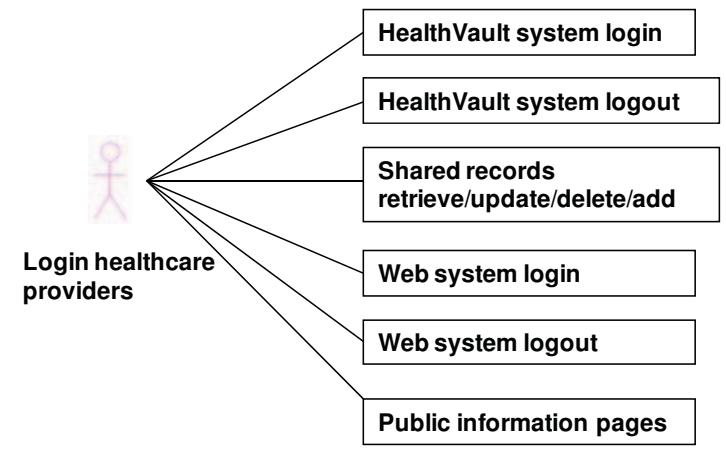

Fig. 7. the basic functionalities for login healthcare providers.

giver's advices. The healthcare providers can login to our system, review the customer's shared information and give the advices. A paying service also can be implemented according to the needs.

The sample system runs on Microsoft windows XP, using an ASP.NET[24] platform and IIS web server. It can be run on the main web browsers, such as Internet Explorer 7+ and Firefox2+. Secure Socket Layer (SSL)[25] service[26] also can be easily set up through IIS for some pages related user private information, which is expected to be security-enhanced.

This sample information system (IS) was developed on Windows XP + SP2 + Visual Studio .NET 2005 + HealthVault Development Kit. ASP.NET/Javascript and C\# are the web programming languages to deal with the web programming. All the HealthVault API will be programmed in $\mathrm{C} \#$ and integrated into the related web pages.

Several public healthcare information pages including, such as, home page, services page, about us and contact us, to provide some general healthcare information should be available to the public for free. Some features, mainly used by login users to access to the Web-enabled online health care service are the following: (1) User Login to our sample system/HealthVault system; (2) Customer Retrieve/Update/Delete/Add the his/her health information in HealthVault; (3) Providers Retrieve/Add information to a specific customer's health record; and (4) Logout from HealthVault system, Logout from our service.

\section{CONCLUSIONS}

HealthVault based Web-enabled healthcare service solution is a novel e-healthcare solution to deal with the quickly increasing e-Paitent's needs. With security-enhanced and privacy-enhanced HealthVault system in the back-end, this type of applications provides not only a time effective, cost 


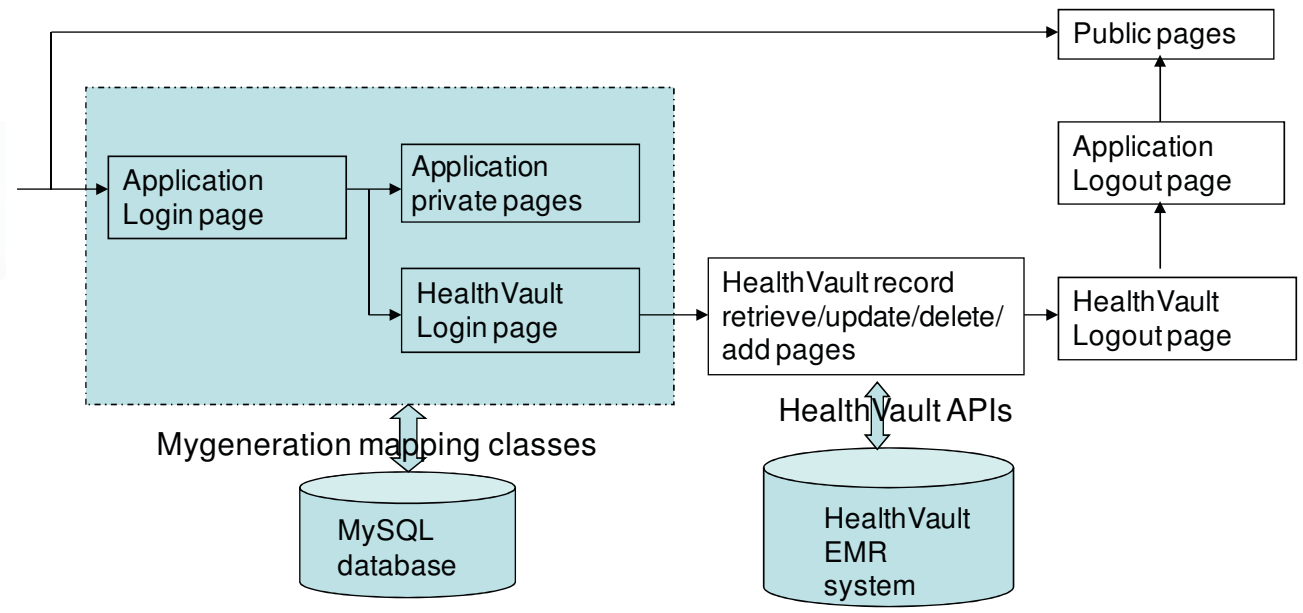

Fig. 4. The sample system architecture.

effective but also interoperable, secure and well-performed solution for those independent healthcare providers.In this paper, we present the general requirements, system design and its capability in rapid development and low cost. The interoperability, security and performance is mostly depended on the back-end HealthVault system but not discussed and evaluated in this paper.Therefore, to investigate the performance and scalability of such a XML database in a highly concurrent circumstance will be our and Microsoft's future work.

\section{REFERENCES}

[1] J. Rodrigues and B. Vaidya, "Health Information Systems: Concepts, Methodologies, Tools and Applications," in multi- volume publication Health Information Systems: Concepts, Methodologies, Tools and Applications, IGI Global, 2010.

[2] http://www.hc-sc.gc.ca/

[3] http://www.infoway-inforoute.ca/

[4] D. Goldstein, "E-healthcare: harness the power of internet e-commerce \& e-care," AN ASPEN PUBLICATION, ISBN 0-8342-1365-6.

[5] G. Yang,"Body Sensor Networks", Springer, ISBN: 978-1-84628-272-0

[6] http://www.physiciansnew.com/

[7] http://msdn.microsoft.com/en-us/healthvault/default.aspx

[8] http://www.oscarcanada.org/

[9] "Broad issues to address in the successful implementation of an Electronic Health Records system" e-Mzanzi Information Society, http://www.pnc.gov.za/, vol. 3, No. 07.

[10] T.D. Gunter, and N.P. Terry, "The Emergence of National Electronic Health Record Architectures in the United States and Australia: Models, Costs, and Questions," Journal of Medical Internet Research, vol. 7, No. $1,2005$.

[11] http://MITRE: center for enterprise modernization: electronic health records overview/

[12] http://www.annals.org/cgi/content/

[13] "The Clinical Systems Hospital IT Market" Himss Foundation, 19982005.

[14] S. Grace, "The Road to EMR Interoperability" http://www.physicianspractice.com/, Nov 2009.

[15] http://www.eclinicalworks.com/

[16] http://www.medcomsys.com/

[17] http://www.medinformatix.com/

[18] http://www.himss.org/foundation/histdata.asp/

[19] http://www.health.gov.bc.ca/socsec/

[20] http://www.iis.net/

[21] http://msdn.microsoft.com/en-us/healthvault/default.aspx/

[22] http://www.mysql.com/why-mysql/topreasons.html

[23] http://www.mygenerationsoftware.com/portal/default.aspx/

[24] http://www.asp.net
[25] "SSL Protocol: Version 3.0," Netscape's final SSL 3.0 draft , November 1996.

[26] http://www.microsoft.com/technet/prodtechnol/WindowsServer2003/Library/ IIS/56bdf977-14f8-4867-9c51-34c346d48b04.mspx?mfr=true/ 